\title{
Restricted and Repetitive Behaviour in Persons with Autism (Ages 0-18): An Integrative Review of Treatment Related to Occupational Therapy
}

\author{
Melissa Patriquin*, Diane MacKenzie and Joan Versnel
}

Dalhousie University, Halifax Nova Scotia, Canada

\begin{abstract}
Objective: To identify evidence-based behavioural interventions used to decrease restricted and repetitive behaviour (RRB) in children with autism (ages 0-18); to understand the application of these strategies within the PEO (Person, Environment, Occupation) Model of Occupational Performance and the role of the occupational therapist in addressing this limitation

Background: RRB is a core feature of autism that often impedes functional behaviour. Decreasing RRB to enable functional behaviours is central to the occupational therapist's role with this population. Many interventions identified as effective for this impairment are based on the principles of applied behaviour analysis (ABA)

Method: An integrative review of the literature was completed to identify interventions targeting RRBs. Those found to be effective are described within an ABA framework and within the PEO Model of Occupational Performance.

Results: This review identified twenty-eight effective interventions used to treat RRBs in autism relevant to the field of occupational therapy. Categorization of interventions using an ABA framework and the PEO Model allowed comparison between approaches and application to occupational therapy practice.

Conclusion: A functional behaviour approach (FBA) used in combination with the PEO model will enable greater understanding of RRBs and provide a more comprehensive approach to the treatment of RRBs in children with autism.
\end{abstract}

Keywords: Autism, repetitive behaviour, occupational therapy, PEO model, behavioural intervention.

\section{INTRODUCTION}

One of the primary criteria for the diagnosis of autism spectrum disorder (ASD) is the presence of restricted and repetitive patterns of behaviour (RRBs) [1] Impairments included along the continuum of RRBs may include stereotypical or repetitive motor movements such as continuous jumping or spinning, repetitive use of objects (playing with only one toy for a prolonged length of time), speech or speech sounds (repeating the same word or sound over a prolonged length of time), insistence on sameness such as wanting to do things in the same way and having difficulty with changes in routine, highly restricted interests (only talking about or playing with items related to the same topic or theme with little variance), as well as hyper or -hypo reactivity to sensory input (an over-reaction or under-reaction to sensory stimuli) or an unusual interest in sensory aspects of the environment [1].

RRBs can potentially cause significant functional impairment in the daily lives of children with autism [2]. RRBs may cause an individual to stand out by making them look and or act differently, potentially affecting social interactions, personal relationships, and possibly

*Address correspondence to this author at the Dalhousie University, Halifax Nova Scotia, Canada; Tel: 902-754-5891; Fax: 902-928-0297.

E-mail: mmaclell@dal.ca leading to social stigmatism and social isolation [3]. In addition, rather than participating in or choosing to perform tasks (occupations) that are typically expected of children their age, children with autism often participate in activities that may not be considered functional or purposeful such as RRBs. By repetitively performing the same seemingly non-purposeful behaviours these individuals often do not have the opportunity to learn more functional behaviours and acquire skills needed for expected or novel tasks or occupations.

Occupational therapy is concerned with promoting health and well-being through engagement in occupation [4]. Occupations include activities or tasks that are considered necessary for daily living [5]. Occupations also include those tasks or activities that are important or meaningful to the person them self [5]. For individuals between the ages of 0-18 typical, age appropriate, or expected occupations may include such activities as progressive independence in self-care tasks such as dressing, eating, and toileting [5]. Leisure occupations may include such things as play with toys, playing/interacting with friends, perhaps sports, crafts or other hobbies [5]. Progressive independence in productivity occupations could include school work, chores, and/or eventually paid employment [5]. There are sometimes barriers to functional performance in meaningful activities. An occupational therapist will aim 
to help their clients overcome occupational dysfunction by enhancing skills, modifying the environment, and/or altering the occupation.

The Person Environment Occupation (PEO) model of occupational performance [6] is a practice framework used by occupational therapists to understand the relationship and interactions between the person, the environment, and occupation. The PEO model proposes that the characteristics of the three constructs interact to determine an individual's occupational performance in everyday function. Dysfunctional occupational performance can result when there is not a good fit between these constructs. Occupational therapists aim to maximize occupational performance and decrease dysfunction in tasks by influencing change in any, some, or all of these components [6].

RRB's often act as barriers to functional performance. These barriers may occur secondary to limitations at the 'person' level within the PEO model. For example, perhaps children with autism engage in RRBs secondary to limited physical, cognitive, or perceptual skills required to perform more functional behaviors. The barrier to functional performance may also be 'occupation' based. For example, the child with autism may have a stronger preference or have developed a habit for the RRB. Participation in that RRB may then take the place of participation in a more meaningful task or occupation. Engagement in occupation may also be limited as a result of the environment in which the occupation is being performed in. Perhaps the physical or sensory aspects of the child's environment are acting as barriers to functional performance in that task or occupation.

Given the potential impact RRBs have on the functional ability and quality of life for children with autism (ages 0-18), occupational therapists have an important role to play with this population. All professionals working with this diagnostic group must be vigilant that the interventions implemented are evidence-based, efficient and effective. A clear understanding of effective interventions to address these concerns is therefore warranted.

Many evidence-based interventions used to address RRBs have been developed based on the field of Applied Behavioural Analysis (ABA). An ABA approach considers the relationship between behaviour and the environment: what happens before the behaviour occurs, that may trigger the behaviour (antecedent) and what happens after the behaviour occurs (the consequences) potentially reinforces the behaviour. Specifically, the use of Functional Behavioural Assessment (FBA) has allowed a greater understanding of why individuals perform the behaviours they do, including RRB.

Boyd, Mcdonough, \& Bodfish [7] gathered information regarding a variety of approaches used to treat RRB and classified them using an ABA framework. Understanding these behavioural interventions through the lens of the PEO model [6] will relate these treatments to occupational therapy practice. The purpose of this paper is to identify effective interventions used by clinicians to manage RRB in children diagnosed with autism and gain an understanding of how these interventions relate to the role of the occupational therapist using the PEO model.

\section{MATERIALS AND METHODS}

This integrative review based on Whittemore \& Knafl's [8] approach, aimed to collect and critically examine relevant information regarding effective interventions used to decrease the occurrence of RRBs within an ABA /FBA framework and then apply this understanding to occupational therapy using the PEO model [6], This method allowed direct comparison between both frames of reference. An integrative review includes applicable empirical and theoretical literature that provided a comprehensive understanding of the related concepts [8].

\section{Search Strategy}

Six electronic databases were searched (Cinahl, ERIC, Proquest Nursing and Allied Health, PubMed, OTSeeker, and PsycINFO) for English language articles published between 2005 and 2015 to ensure the most recent information was examined. Search terms used for this review included autism, treatment/intervention, and repetitive behaviour/stereotypical behaviour. Inclusion criteria specified only studies that used human subjects ranging in age from $0-18$. The search source types included scholarly articles, journal articles, and books. Qualitative, quantitative, and mixed methodology studies were considered as well as systematic and scoping reviews.

Studies were excluded upon review of the abstracts if the treatment was not relevant to the scope of practice of occupational therapy (i.e. treatment such as use of medication to decrease repetitive behaviour), if 
treatment was not clearly defined, and if restricted or repetitive behaviour was not a dependent variable. Additional exclusion criteria included articles in which study participants were not diagnosed with autism and if all study subjects were older than eighteen. The search and selection process is illustrated in Figure 1.

\section{RESULTS}

\section{Article Selection and Quality Appraisal}

After initial searches and review of article abstracts to ensure all inclusion and exclusion criteria were met, fourteen publications, two review papers and twelve individual intervention studies, were included in this review. Search results are presented in Table 1 and organized into two categories for clarity: 1) reviews and 2) intervention studies. All were initially appraised under the following headings: study information; (author/year of publication); type of study (design); sample (size, diagnosis, and ages); the type of intervention; and results of the intervention (e.g., effective or not at decreasing RRB).

From the fourteen publications, only the original study or review paper that reported results of effective

\footnotetext{
14 Pubmed (Autism+RRB* + intervention)

56 Cinahl (Autism $+\mathrm{RRB}^{*}+$ intervention or treatment)

11 ERIC (Autism $+\mathrm{RRB}^{*}+$ intervention or stereotypical)

20 Proquest Nursing and Allied Health,

0 OTSeeker (Autism, RRB* or stereotypical and intervention

88 PsycINFO (Autism, stereotypical or RRB*, and intervention)

189: TOTAL Number of publication identified by search
}

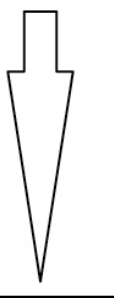

175 - Number of articles excluded based on titles and abstracts review and duplication from other databases. If intervention used was not relevant to the field of occupational therapy, if treatment was not clearly defined, if RRB was not a dependent variable measured as a result of implementing the intervention, if subjects were not formally diagnosed with autism, or if not all subjects were within the age range $0-18$ article was excluded

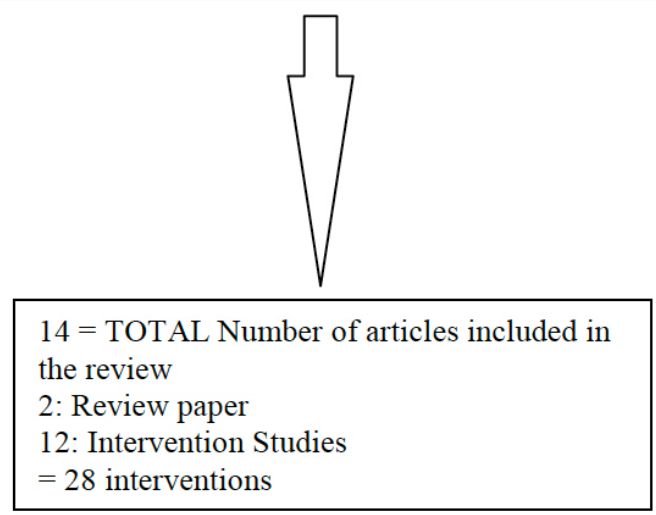

*RRB (Restricted and Repetitive Behaviour)

Figure 1: Search and Selection Process. 
Table 1: Summary of Articles Included in the Integrative Review

\begin{tabular}{|c|c|c|c|c|c|}
\hline $\begin{array}{c}\text { Study } \\
\text { Information }\end{array}$ & $\begin{array}{l}\text { Type of } \\
\text { study }\end{array}$ & Sample & Intervention & \multicolumn{2}{|c|}{$\begin{array}{c}\text { Results: } \\
\text { Effectively or ineffectively decrease } \\
\text { RRBs }\end{array}$} \\
\hline \multicolumn{6}{|c|}{ I Reviews } \\
\hline \multirow[t]{11}{*}{$\begin{array}{l}\text { Boyd BA, } \\
\text { Mcdonough } \\
\text { SG, Bodfish JW } \\
\text { 2012. [7] }\end{array}$} & \multirow[t]{11}{*}{$\begin{array}{c}\text { Review } \\
\text { 1974- } 2010\end{array}$} & \multirow[t]{11}{*}{$\begin{array}{l}27 \text { studies } \\
\text { Dx: } \\
\text { ASD * with } \\
\text { RRBs** } \\
\text { Age: Does } \\
\text { not specify }\end{array}$} & $\begin{array}{l}\text { Response Interruption and Redirection } \\
\text { /response blocking (Physically or verbally } \\
\text { blocking from engaging in behaviour) }\end{array}$ & $\begin{array}{c}\text { Ahearn et al. } 2007 . \\
\text { [9] } \\
\text { Koegal et al. } 1974 \\
\text { [10] } \\
\text { Liu-Gitz L, Banda } \\
\text { DR 2010. [11] }\end{array}$ & \\
\hline & & & $\begin{array}{l}\quad \text { Response cost procedures } \\
\text { (Removal of a positive consequence } \\
\text { when a repetitive behaviour occurs) }\end{array}$ & $\begin{array}{l}\text { Athens et al. } 2008 . \\
\text { [12] } \\
\text { Sidener et al. } \\
\text { 2005. [13] }\end{array}$ & \\
\hline & & & $\begin{array}{l}\text { Consequence based uses Circumscribed } \\
\text { Interests }(\mathrm{Cl}) \\
\text { (Cl used as a contingent reinforcer } \\
\text { delivered on the occurrence of } \\
\text { appropriate behaviours) }\end{array}$ & $\begin{array}{l}\text { Charlop-Christy M, } \\
\text { Haymes L. } \\
\text { 1996[15] 1998) } \\
\text { [16] }\end{array}$ & \\
\hline & & & $\begin{array}{l}\text { Differential reinforcement of variability } \\
\text { (Reinforcing the individual for varying } \\
\text { behavioural responses with the } \\
\text { reinforcement being linked to how novel } \\
\text { the behaviour is) }\end{array}$ & $\begin{array}{l}\text { Boyd et al. } 2010 \\
\quad[17] \\
\text { Miller, Neuringer } \\
2000[18]\end{array}$ & \\
\hline & & & $\begin{array}{l}\text { Visual or verbal cues } \\
\text { (Cues used to forewarn the child or allow } \\
\text { engagement in calming or highly preferred } \\
\text { task prior to difficult task) }\end{array}$ & $\begin{array}{l}\text { Conroy et al. } 2005 \\
\text { [19] Horner et al. } \\
1997 \text { [20] }\end{array}$ & \\
\hline & & & $\begin{array}{l}\text { Antecedent Based uses of Circumscribed } \\
\text { interests }(\mathrm{Cl}) \\
(\mathrm{Cl} \text { is embedded in the task the person } \\
\text { will engage in to increase desired } \\
\text { behaviour during the activity }\end{array}$ & $\begin{array}{l}\text { Baker,2000. [24] } \\
\text { Baker et al. } 1998 . \\
\text { [25] } \\
\text { Boyd et al. } 2007 . \\
\quad[26]\end{array}$ & \\
\hline & & & $\begin{array}{l}\text { Visual schedules or video based } \\
\text { technologies } \\
\text { (used to tolerate change or expand } \\
\text { repetitive behaviour) }\end{array}$ & $\begin{array}{l}\text { Hine, Wolery } 2006 \\
\text { [27] } \\
\text { Odom et al. } 2003 . \\
{[28]}\end{array}$ & \\
\hline & & & $\begin{array}{c}\text { Cognitive Behaviour Therapy/Exposure } \\
\text { Response Prevention }\end{array}$ & $\begin{array}{l}\text { Lehmkuhl et al. } \\
2008^{\star * * *}[29]\end{array}$ & \\
\hline & & & $\begin{array}{l}\text { (Cognitive reframing and exposure } \\
\text { response prevention exercises) }\end{array}$ & $\begin{array}{l}\text { Reaven, Hepburn } \\
\text { 2003. [30] }\end{array}$ & \\
\hline & & & $\begin{array}{l}\text { Functional Communications training } \\
\text { (teaching appropriate communication } \\
\text { responses that can be used to obtain the } \\
\text { same reinforcer) }\end{array}$ & $\begin{array}{l}\text { Kennedy et al. } \\
\text { 2000. [31] }\end{array}$ & \\
\hline & & & $\begin{array}{l}\text { Physical Exercise } \\
\text { (participates prior to subsequent activity } \\
\text { associated with repetitive behaviour) }\end{array}$ & $\begin{array}{c}\text { Kern et al. } 1984 . \\
\text { [32] }\end{array}$ & \\
\hline
\end{tabular}


(Table 1). Continued.

\begin{tabular}{|c|c|c|c|c|c|}
\hline $\begin{array}{c}\text { Study } \\
\text { Information }\end{array}$ & $\begin{array}{l}\text { Type of } \\
\text { study }\end{array}$ & Sample & Intervention & \multicolumn{2}{|c|}{$\begin{array}{c}\text { Results: } \\
\text { Effectively or ineffectively decrease } \\
\text { RRBs }\end{array}$} \\
\hline \multirow{9}{*}{$\begin{array}{l}\text { Patterson SY, } \\
\text { Smith V, Jelen } \\
\text { M. 2010. [33] }\end{array}$} & \multirow{9}{*}{$\begin{array}{c}\text { Systematic } \\
\text { Review } \\
10 \text { single } \\
\text { case studies } \\
\text { Studies up } \\
\text { until June } \\
2008\end{array}$} & \multirow{9}{*}{$\begin{array}{c}\text { Size: } 17 \\
\text { participants } \\
\text { Dx: ASD* } \\
\text { Age } \\
2 y 11 \mathrm{mo}- \\
\text { 26years }\end{array}$} & $\begin{array}{l}\text { Response interruption and redirection } \\
\text { (RIRD) }\end{array}$ & $\begin{array}{l}\text { Ahearn et al. } \\
\text { 2007[9] }\end{array}$ & \\
\hline & & & $\begin{array}{l}\text { Differential reinforcement and extinction } \\
\text { (reinforcement in the event of a correct } \\
\text { response, and no reinforcement when } \\
\text { there is not a correct response) }\end{array}$ & $\begin{array}{c}\text { Rehfeldt, } \\
\text { Chambers, } 2003 . \\
{[34]}\end{array}$ & \\
\hline & & & Scheduled thinning of response blocking & & $\begin{array}{l}\text { Tarbox et al. } 2002 . \\
{[35]}\end{array}$ \\
\hline & & & $\begin{array}{l}\text { Noncontingent Reinforcement and } \\
\text { response blocking } \\
\text { (the delivery of reinforcers according to a } \\
\text { schedule that is not response contingent } \\
\text { and blocking the response before it starts) }\end{array}$ & & Carr et al. 2002 [36] \\
\hline & & & $\begin{array}{l}\text { Noncontingent Reinforcement and } \\
\text { response interruption (RI) } \\
\text { (the delivery of reinforcers according to a } \\
\text { schedule that is not response contingent } \\
\text { and interrupting the behaviour as it is } \\
\text { occurring) }\end{array}$ & & $\begin{array}{l}\text { Cicero } 2007 \text { [37] } \\
\text { (No more difference } \\
\text { than RI alone) }\end{array}$ \\
\hline & & & $\begin{array}{l}\text { Noncontingent access } \\
\text { (Continuous access, access to items not } \\
\text { dependent on behaviour) }\end{array}$ & $\begin{array}{c}\text { Roane et al. } 2003 . \\
\text { [38] }\end{array}$ & \\
\hline & & & $\begin{array}{l}\text { Matched stimulation and noncontingent } \\
\text { reinforcement } \\
\text { (Access to items that produced similar } \\
\text { sounds to the problematic behaviour, } \\
\text { Access not contingent on behaviour) }\end{array}$ & $\begin{array}{c}\text { Rapp } 2007 . \\
\text { [39] }\end{array}$ & \\
\hline & & & $\begin{array}{l}\text { Functional communication training } \\
\text { (teaching appropriate communication } \\
\text { responses that can be used to obtain the } \\
\text { same reinforcer) }\end{array}$ & & $\begin{array}{c}\text { Kennedy et al. } 2000 . \\
{[31]}\end{array}$ \\
\hline & & & $\begin{array}{l}\text { Antecedent based visual cue card } \\
\text { strategy } \\
\text { (Visual prompts provided before the } \\
\text { behaviour occurs) }\end{array}$ & & $\begin{array}{c}\text { Conroy et al. } 2005 \\
\text { [19] }\end{array}$ \\
\hline \multicolumn{6}{|c|}{ II Intervention Studies } \\
\hline $\begin{array}{l}\text { Boyd BA, } \\
\text { McDonough } \\
\text { SG, Rupp B, } \\
\text { Khan F, } \\
\text { Bodfish JW. } \\
\text { 2011. [41] }\end{array}$ & $\begin{array}{l}\text { Multiple } \\
\text { Single case } \\
\text { design }\end{array}$ & $\begin{array}{l}\text { Size: } 5 \\
\text { Dx: ASD* } \\
\text { Age: } 48 \\
\text { months }\end{array}$ & $\begin{array}{c}\text { Family-Implemented Treatment for } \\
\text { Behavioural Inflexibility } \\
12 \text { week direct instruction to teach parents } \\
\text { to } \\
\text { identify environmental cues that elicited } \\
\text { RRBs }^{* *} \text {, } \\
\text { how to inhibit repetitive behaviours in the } \\
\text { presence of these cues and replace them } \\
\text { with alternative, adaptive behaviours }\end{array}$ & $\begin{array}{l}\text { Decrease in } \\
\text { repetitive } \\
\text { behaviours }\end{array}$ & \\
\hline
\end{tabular}


(Table 1). Continued.

\begin{tabular}{|c|c|c|c|c|c|}
\hline \multirow[t]{2}{*}{$\begin{array}{c}\text { Study } \\
\text { Information }\end{array}$} & \multirow[t]{2}{*}{$\begin{array}{l}\text { Type of } \\
\text { study }\end{array}$} & \multirow[t]{2}{*}{ Sample } & \multirow[t]{2}{*}{ Intervention } & \multicolumn{2}{|c|}{$\begin{array}{c}\text { Results: } \\
\text { Effectively or ineffectively decrease } \\
\text { RRBs }\end{array}$} \\
\hline & & & & Effective & Ineffective \\
\hline $\begin{array}{l}\text { Boyd BA, } \\
\text { Woodard CR, } \\
\text { Bodfish JW. } \\
\text { 2013. [42] }\end{array}$ & $\begin{array}{l}\text { Multiple } \\
\text { single case } \\
\text { design }\end{array}$ & $\begin{array}{c}\text { Size: } 5 \\
\text { Dx: ASD* and } \\
\text { co-morbid } \\
\text { intellectual } \\
\text { disabilities } \\
\text { Lived in a } \\
\text { residential } \\
\text { facility } \\
\text { Age: School } \\
\text { age (5-11) }\end{array}$ & $\begin{array}{l}\text { Exposure response prevention } \\
\text { (Exposure: repeated gradual exposure to } \\
\text { environmental stimuli that are associated } \\
\text { with anxiety and subsequent compulsive } \\
\text { behaviour) } \\
\text { AND } \\
\text { Response prevention (active avoidance of } \\
\text { the compulsive act) } \\
10 \text { sessions }\end{array}$ & $\begin{array}{l}\text { Decreased lower } \\
\text { order RBs } \\
\text { Most effective } \\
\text { when there are } \\
\text { tangible } \\
\text { antecedent stimuli. } \\
\text { that reliably predict } \\
\text { the onset of RB }{ }^{* * *}\end{array}$ & \\
\hline $\begin{array}{c}\text { Grahame V, } \\
\text { Brett D, Dixon } \\
\text { L, McConachie } \\
\text { H, Lowry J, } \\
\text { Rodgers J, } \\
\text { Couteur A. } \\
\text { 2015. [43] }\end{array}$ & $\begin{array}{c}\text { RCT } \\
\text { Intervention } \\
\text { vs delayed } \\
\text { intervention }\end{array}$ & $\begin{array}{l}45 \text { families } \\
\text { Parents of } \\
\text { children with } \\
\text { ASD* } \\
\text { Aged 3-7 } \\
\text { years }\end{array}$ & $\begin{array}{c}\text { Managing Repetitive Behaviours Program } \\
\text { (Parent-group intervention: } \\
\text { Psycho-educational intervention } \\
\text { incorporates knowledge of } \\
\text { ASD, the principles of a Functional } \\
\text { analysis approach } \\
\text { Combined with the mutual support of } \\
\text { group peer learning) }\end{array}$ & $\begin{array}{l}\text { Parent-reported } \\
\text { changes in RRBs* } \\
\text { vs delayed group }\end{array}$ & \\
\hline $\begin{array}{l}\text { Kang S, } \\
\text { O'Reilly M, } \\
\text { Rojeski L, } \\
\text { Blenden K, Xu } \\
\text { Z, Davis T, } \\
\text { Lancioni G. } \\
2013 . \\
\text { [44] }\end{array}$ & $\begin{array}{c}\text { Multiple } \\
\text { single case } \\
\text { design } \\
\text { ABA design }\end{array}$ & $\begin{array}{l}\text { Size: } 3 \\
\text { Dx: ASD* } \\
\text { Age: } 3-8 \\
\text { years }\end{array}$ & $\begin{array}{c}\text { Tangible reinforcer } \\
\text { Compared to Social Reinforcement by } \\
\text { others }\end{array}$ & $\begin{array}{l}\text { When social praise } \\
\text { used as the } \\
\text { reinforcer }\end{array}$ & \\
\hline $\begin{array}{l}\text { Kuhn DE, } \\
\text { Hardesty SL, } \\
\text { Sweeney NM. } \\
\text { 2009. [45] }\end{array}$ & $\begin{array}{c}\text { Single Case } \\
\text { study } \\
\text { ABAB } \\
\text { Design }\end{array}$ & $\begin{array}{l}\text { Size: } 1 \\
\text { Dx: } \text { ASD* and }^{*} \\
\text { moderate } \\
\text { mental } \\
\text { retardation } \\
\text { Age: } 16 \text { years }\end{array}$ & $\begin{array}{l}\text { Functional communication training with } \\
\text { extinction of destructive behaviour and } \\
\text { response blocking of repetitive } \\
\text { straightening (physically or mechanically } \\
\text { disrupting the response before its } \\
\text { completion). }\end{array}$ & $\begin{array}{l}\text { Effective when all } \\
\text { three approaches } \\
\text { were used } \\
\text { together }\end{array}$ & \\
\hline $\begin{array}{l}\text { Lehmkuhl HD, } \\
\text { Storch EA, } \\
\text { Bodfish JW, } \\
\text { Geffken GR. } \\
\text { 2008. [29] }\end{array}$ & $\begin{array}{l}\text { Single case } \\
\text { study }\end{array}$ & $\begin{array}{l}\text { Size; } 1 \\
\text { Dx ASD* and } \\
\text { Obsessive } \\
\text { Compulsive } \\
\text { Disorder } \\
\text { (OCD) } \\
\text { Age: } 12 \text {-year- } \\
\text { old male }\end{array}$ & $\begin{array}{l}\text { Cognitive behavioural therapy } \\
\text { with exposure and response prevention. }\end{array}$ & $\begin{array}{l}\text { Cognitive } \\
\text { behavioural } \\
\text { therapy is effective } \\
\text { in reducing } \\
\text { OCD symptoms in } \\
\text { a child with Autism }\end{array}$ & \\
\hline $\begin{array}{c}\text { Loftin RL, } \\
\text { Odom SL, } \\
\text { Lantz JF 2008. } \\
\text { [46] }\end{array}$ & $\begin{array}{l}\text { Multiple } \\
\text { single case } \\
\text { multiple } \\
\text { baseline } \\
\text { design }\end{array}$ & $\begin{array}{c}\text { Size: } 3 \\
\text { Dx: } \text { ASD* }^{*} \\
\text { Age: } 9,10,10\end{array}$ & $\begin{array}{l}\text { Multi-component social skills intervention } \\
\text { (including peer training, social initiation } \\
\text { instruction, and self-monitoring) }\end{array}$ & $\begin{array}{l}\text { Participants' } \\
\text { repetitive motor } \\
\text { behaviour was } \\
\text { reduced. } \\
\text { Changes in social } \\
\text { behaviour and in } \\
\text { repetitive motor } \\
\text { behaviour } \\
\text { maintained more } \\
\text { than one month } \\
\text { after the } \\
\text { intervention ended }\end{array}$ & \\
\hline
\end{tabular}


(Table 1). Continued.

\begin{tabular}{|c|c|c|c|c|c|}
\hline $\begin{array}{c}\text { Study } \\
\text { Information }\end{array}$ & $\begin{array}{l}\text { Type of } \\
\text { study }\end{array}$ & Sample & Intervention & \multicolumn{2}{|c|}{$\begin{array}{c}\text { Results: } \\
\text { Effectively or ineffectively decrease } \\
\text { RRBs }\end{array}$} \\
\hline $\begin{array}{l}\text { Love JJ, Miguel } \\
\text { CF, Fernand J } \\
\text { K, LaBrie JK. } \\
\text { 2012. [3] }\end{array}$ & $\begin{array}{l}\text { Case study } \\
\text { Quantitative } \\
\text { And } \\
\text { qualitative }\end{array}$ & $\begin{array}{l}\text { Size: } 2 \\
\text { Dx: ASD* } \\
\text { Age: } 9,10\end{array}$ & $\begin{array}{l}\text { Response interruption and redirection } \\
\text { (RIRD) } \\
\text { Noncontingent access to matched } \\
\text { stimulus (MS) } \\
\text { Response interruption and redirection } \\
\text { AND Noncontingent access to matched } \\
\text { stimulus }\end{array}$ & $\begin{array}{l}\text { Client 1: Similar } \\
\text { suppressive } \\
\text { effects on vocal } \\
\text { stereotypy across } \\
\text { treatment } \\
\text { conditions. } \\
\text { Client 2: Slightly } \\
\text { greater } \\
\text { suppression of } \\
\text { stereotypy was } \\
\text { associated with } \\
\text { MS and RIRD } \\
\text { together. }\end{array}$ & \\
\hline $\begin{array}{l}\text { Rodriguez NM, } \\
\text { Thompson RH, } \\
\text { Stocco CS, } \\
\text { Schlichenmeyer } \\
\text { K. 2013. [48] }\end{array}$ & $\begin{array}{c}\text { Multiple } \\
\text { single case } \\
\text { ABA } \\
\text { design? }\end{array}$ & $\begin{array}{c}\text { Size: } 3 \\
\text { Dx: ASD* and } \\
\text { demonstrating } \\
\text { ordering and } \\
\text { arranging that } \\
\text { was } \\
\text { problematic } \\
\text { Age: } 9-14 \text { yrs. }\end{array}$ & $\begin{array}{l}\text { Matched Item plus blocking } \\
\text { Matched Item plus prompts } \\
\text { plus blocking }\end{array}$ & $\begin{array}{l}\text { Depending on the } \\
\text { function of the } \\
\text { behaviour } \\
\mathrm{RB}^{* * *} \text { decreased } \\
\text { when the function } \\
\text { of the behaviour } \\
\text { was determined } \\
\text { and treatment was } \\
\text { chosen based on } \\
\text { the FBA }\end{array}$ & \\
\hline $\begin{array}{c}\text { Watling RL, } \\
\text { Dietz J. } 2007 . \\
\text { [50] }\end{array}$ & $\begin{array}{l}\text { Single- } \\
\text { subject } \\
\text { study used } \\
\text { an ABAB } \\
\text { design }\end{array}$ & $\begin{array}{c}\text { Size: } 4 \\
\text { Dx: ASD* } \\
\text { Age: } 3-4.4 \\
\text { years }\end{array}$ & $\begin{array}{c}\text { Ayres Sensory Integration } \\
\text { Vs. play scenario }\end{array}$ & & $\begin{array}{c}\text { No difference } \\
\text { between } 2 \text { groups on } \\
\mathrm{RB}^{* \star *}\end{array}$ \\
\hline
\end{tabular}

Notes:

Dx = Diagnosis

*(ASD) Autism Spectrum Disorder.

${ }^{* *}(\mathrm{RRB})$ Restricted and Repetitive Behaviours.

***(RB) Restricted Behaviours.

${ }^{* * * *}$ Studies are also listed in intervention studies.

interventions to decrease RRBs is included in Table 2. Those interventions found to be ineffective or inconclusive (i.e., had no effect on RRB or had no more effect compared to treatment as usual) by the respective authors or by the authors of the reviews were not included. All of the interventions noted in the review by Boyd et al. [7] were identified as effective in the reduction of RRBs. Only some of the interventions 
Table 2: Classification and Analysis of Effective RRB Interventions that Target Person, Environment or Occupation

\begin{tabular}{|c|c|c|c|}
\hline $\begin{array}{l}\text { Study Information: } \\
\text { Author (year of publication) }\end{array}$ & Intervention & $\begin{array}{l}\text { Intervention Category } \\
\text { (Boyd et al. 2012) }\end{array}$ & $\begin{array}{l}\text { PEO*Level of Treatment } \\
\quad \text { (Law et al. 1996) }\end{array}$ \\
\hline \multicolumn{4}{|c|}{ Treatment Approaches that Target the Environment } \\
\hline $\begin{array}{c}\text { Conroy et al. } 2005 \text { [19] } \\
\text { \& Horner et al. } 1997 \text { [20] } \\
\text { In: Boyd, BA, Mcdonough, SG, } \\
\text { Bodfish JW. 2012. [7] }\end{array}$ & $\begin{array}{l}\text { Visual or verbal cues (to forewarn the child } \\
\text { or allow engagement in calming or highly } \\
\text { preferred task prior to difficult task) }\end{array}$ & $\begin{array}{l}\text { Antecedent: modifying } \\
\text { environment }\end{array}$ & Environment \\
\hline $\begin{array}{l}\text { Azrin et al. 1988. [14] } \\
\text { In: Boyd BA, Mcdonough SG, } \\
\text { Bodfish JW. 2012. [7] }\end{array}$ & $\begin{array}{l}\text { Differential reinforcement } \\
\text { (Reinforce other behaviour the individual } \\
\text { displays) }\end{array}$ & Consequence & Environment \\
\hline $\begin{array}{c}\text { Athens et al. 2008[12] } \\
\text { Sidener et al. 2005[13] } \\
\text { In: Boyd BA, Mcdonough SG, } \\
\text { Bodfish JW. 2012. [7] }\end{array}$ & $\begin{array}{l}\text { Response cost procedures: removal of a } \\
\text { positive consequence when a repetitive } \\
\text { behaviour occurs }\end{array}$ & Consequence & Environment \\
\hline $\begin{array}{l}\text { Boyd BA, Woodard CR, } \\
\text { Bodfish JW. 2013. [42] }\end{array}$ & $\begin{array}{l}\text { Exposure response prevention: ERP } \\
\text { (Exposure: repeated gradual exposure to } \\
\text { environmental stimuli that are associated } \\
\text { with anxiety and subsequent compulsive } \\
\text { behaviour) } \\
\text { (Response prevention: active avoidance of } \\
\text { the compulsive act) }\end{array}$ & $\begin{array}{l}\text { Antecedent: modify } \\
\text { environment and } \\
\text { consequence }\end{array}$ & Environment \\
\hline $\begin{array}{c}\text { Kang S, O'Reilly M, Rojeski L, } \\
\text { Blenden K, Xu Z, Davis T, } \\
\text { Lancioni G. 2013. [44] }\end{array}$ & $\begin{array}{c}\text { Tangible reinforcer } \\
\text { Compared to social reinforcement by others }\end{array}$ & Consequence & Environment \\
\hline $\begin{array}{l}\text { Rehfeldt RA, Chambers MR. } \\
\text { 2003. [34] } \\
\text { In: Patterson SY, Smith V, } \\
\text { Jelen M. 2010. [33] }\end{array}$ & $\begin{array}{l}\text { Differential reinforcement and extinction } \\
\text { (Reinforcement in the event of a correct } \\
\text { response, and no reinforcement when there } \\
\text { is not a correct response }\end{array}$ & Consequence & Environment \\
\hline \multicolumn{4}{|c|}{ Treatment Approaches that Target the Person } \\
\hline & None & None & None \\
\hline \multicolumn{4}{|c|}{ Treatment Approaches that Target Occupation } \\
\hline $\begin{array}{c}\text { Kern et al. } 1984 \text { [32] } \\
\text { In: Boyd BA, Mcdonough SG, } \\
\text { Bodfish JW. 2012. [7] }\end{array}$ & $\begin{array}{l}\text { Physical exercise (child participates prior to } \\
\text { subsequent activity associated with } \mathrm{RB}^{\star *} \text { ) }\end{array}$ & $\begin{array}{l}\text { Antecedent modify } \\
\text { environment (routine) }\end{array}$ & Occupation \\
\hline
\end{tabular}


(Table 2). Continued.

\begin{tabular}{|c|c|c|c|}
\hline $\begin{array}{l}\text { Study Information: } \\
\text { Author (year of publication) }\end{array}$ & Intervention & $\begin{array}{l}\text { Intervention Category } \\
\text { (Boyd et al. 2012) }\end{array}$ & $\begin{array}{l}\text { PEO*Level of Treatment } \\
\text { (Law et al. 1996) }\end{array}$ \\
\hline \multicolumn{4}{|c|}{ Treatment Approaches that Target the Environment and Person } \\
\hline $\begin{array}{l}\text { Lehmkuhl et al. 2008. [29] }^{* * *} \\
\text { Reaven, Hepburn } \\
\text { 2003. [30] } \\
\text { In: Boyd BA, Mcdonough SG, } \\
\text { Bodfish JW. 2012. [7] }\end{array}$ & $\begin{array}{c}\text { Cognitive Behaviour Therapy /Exposure } \\
\text { Response Prevention } \\
\text { (Cognitive reframing and exposure } \\
\text { response prevention exercises) }\end{array}$ & $\begin{array}{l}\text { Antecedent: skill } \\
\text { Antecedent: modifying } \\
\text { environment and } \\
\text { consequence }\end{array}$ & $\begin{array}{l}\text { Person } \\
\text { Environment }\end{array}$ \\
\hline $\begin{array}{c}\text { Lehmkuhl HD, Storch EA, } \\
\text { Bodfish JW, Geffken GR. } 2008 . \\
\text { [29] }\end{array}$ & $\begin{array}{l}\text { Cognitive behavioural therapy with } \\
\text { exposure and response prevention. }\end{array}$ & $\begin{array}{l}\text { Antecedent: skill } \\
\text { Antecedent: modify } \\
\text { environment } \\
\text { and consequence }\end{array}$ & $\begin{array}{l}\text { Person } \\
\text { Environment }\end{array}$ \\
\hline $\begin{array}{c}\text { Storch EA, Arnold EB, Lewin } \\
\text { AB, Nadeau J, Jones AM, De } \\
\text { Nadai AS, Murphy TK. (2012). } \\
\text { [49] }\end{array}$ & Cognitive Behaviour Therapy x 16 weeks & Antecedent: skill & $\begin{array}{l}\text { Environment (parent skills) } \\
\text { Person (child skills) }\end{array}$ \\
\hline \multicolumn{4}{|c|}{ Treatment Approaches that Target the Environment and the Occupation } \\
\hline $\begin{array}{l}\text { Baker MJ. } 2000 \text { [24], Baker et } \\
\quad \text { al. 1998. [25] } \\
\text { In: Boyd BA, Mcdonough, SG, } \\
\text { Bodfish JW. 2012. [7] }\end{array}$ & $\begin{array}{l}\text { Antecedent Based uses of circumscribed } \\
\text { interests }(\mathrm{Cl})(\mathrm{Cl} \text { is embedded in the task } \\
\text { the person will engage in to increase } \\
\text { desired behaviour during the activity) }\end{array}$ & $\begin{array}{l}\text { Antecedents: modifying } \\
\text { environment }\end{array}$ & $\begin{array}{l}\text { Environment and } \\
\text { occupation }\end{array}$ \\
\hline $\begin{array}{c}\text { Piazza et al. 2000. [21] } \\
\text { Rapp, Vollmer. 2005. [22] } \\
\text { Vollmer et al. 1994. [23] } \\
\text { In: Boyd B A, Mcdonough SG, } \\
\text { Bodfish JW. 2012. [7] }\end{array}$ & $\begin{array}{l}\text { Environmental enrichment } \\
\text { strategies (noncontingent access to } \\
\text { appropriate competing sources of } \\
\text { reinforcement) }\end{array}$ & $\begin{array}{l}\text { Antecedent: modifying } \\
\text { environment }\end{array}$ & $\begin{array}{l}\text { Environment and } \\
\text { occupation }\end{array}$ \\
\hline $\begin{array}{l}\text { Charlop-Christy M, Haymes L. } \\
\text { 1996. [15], 1998[16] } \\
\text { In: Boyd BA, Mcdonough, SG, } \\
\text { Bodfish JW. 2012. [7] }\end{array}$ & $\begin{array}{c}\text { Consequence based uses Circumscribed } \\
\text { Interests }(\mathrm{Cl}) \\
(\mathrm{Cl} \text { used as a contingent reinforcer } \\
\text { delivered on the occurrence of appropriate } \\
\text { behaviours })\end{array}$ & Consequence & $\begin{array}{l}\text { Environment and } \\
\text { occupation }\end{array}$ \\
\hline $\begin{array}{c}\text { Ahearn et al. } 2007 \text { [9] } \\
\text { Koegal et al. 1974. [10] } \\
\text { Liu-Gitz L, Banda DR. 2010. } \\
\text { [11] } \\
\text { In: Boyd BA, Mcdonough S.G. } \\
\text { Bodfish JW. 2012. [7] }\end{array}$ & $\begin{array}{l}\text { Response Interruption and } \\
\text { Redirection/response blocking (Physically } \\
\text { or verbally blocking from engaging in } \\
\text { behaviour) }\end{array}$ & Consequence & $\begin{array}{l}\text { Environment and } \\
\text { occupation }\end{array}$ \\
\hline $\begin{array}{l}\text { Love JJ, Miguel C F, Fernand } \\
\text { JK, LaBrie JK. 2012. [3] }\end{array}$ & $\begin{array}{l}\text { Response interruption and redirection } \\
\text { (RIRD) } \\
\text { Noncontingent access to matched } \\
\text { stimulation (MS) } \\
\text { RIRD and MS }\end{array}$ & $\begin{array}{l}\text { Consequence } \\
\text { Antecedent: modify } \\
\text { environment } \\
\text { Consequence and } \\
\text { Antecedent: modify } \\
\text { environment }\end{array}$ & $\begin{array}{l}\text { Environment and } \\
\text { occupation } \\
\text { Environment and } \\
\text { occupation } \\
\text { Environment and } \\
\text { occupation }\end{array}$ \\
\hline $\begin{array}{l}\text { Roane H S, Kelly ML, Fisher } \\
\text { WW. 2003. [38] } \\
\text { In Patterson SY, Smith V, } \\
\text { Jelen M. 2010. [33] }\end{array}$ & Noncontingent access & $\begin{array}{l}\text { Antecedent: modify } \\
\text { environment }\end{array}$ & $\begin{array}{l}\text { Environment and } \\
\text { occupation }\end{array}$ \\
\hline $\begin{array}{l}\text { Ahearn WH, Clark KM, } \\
\text { Macdonald RPF, Chung BI. } \\
\text { 2007. [9] } \\
\text { In: Patterson SY, Smith V, } \\
\text { Jelen M. 2010. [33] }\end{array}$ & Response interruption and redirection & Consequence & $\begin{array}{l}\text { Environment and } \\
\text { occupation }\end{array}$ \\
\hline $\begin{array}{l}\text { Rapp JT. 2007.[39] } \\
\text { In Patterson SY, Smith V, } \\
\text { Jelen M. 2010. [33] }\end{array}$ & $\begin{array}{c}\text { Matched stimulation and Noncontingent } \\
\text { Reinforcement }\end{array}$ & $\begin{array}{l}\text { Antecedent: modify } \\
\text { environment } \\
\text { and consequence }\end{array}$ & $\begin{array}{l}\text { Occupation and } \\
\text { environment }\end{array}$ \\
\hline
\end{tabular}


(Table 2). Continued.

\begin{tabular}{|c|c|c|c|}
\hline $\begin{array}{l}\text { Study Information: } \\
\text { Author (year of publication) }\end{array}$ & Intervention & $\begin{array}{l}\text { Intervention Category } \\
\text { (Boyd et al. 2012) }\end{array}$ & $\begin{array}{l}\text { PEO*Level of Treatment } \\
\text { (Law et al. 1996) }\end{array}$ \\
\hline $\begin{array}{c}\text { Rodriquez NM, Thompson RH, } \\
\text { Schlichenmeyer K.2013. [48] }\end{array}$ & $\begin{array}{c}\text { Matched Item } \\
\text { plus blocking } \\
\text { Matched Item plus prompts plus blocking }\end{array}$ & $\begin{array}{l}\text { Antecedent: modify } \\
\text { environment } \\
\text { and consequence } \\
\text { Antecedent: modify } \\
\text { environment } \\
\text { and consequence }\end{array}$ & $\begin{array}{l}\text { Occupation and } \\
\text { environment } \\
\text { Occupation and } \\
\text { environment }\end{array}$ \\
\hline \multicolumn{4}{|c|}{ Treatment Approaches that Target the Environment, Occupation and Person } \\
\hline $\begin{array}{l}\text { Kennedy et al. 2000. [31] } \\
\text { Boyd BA, Mcdonough SG, } \\
\text { Bodfish JW. 2012. [7] }\end{array}$ & $\begin{array}{l}\text { Functional communications training } \\
\text { (teaching appropriate communication } \\
\text { responses that can be used to obtain the } \\
\text { same reinforcer) }\end{array}$ & $\begin{array}{l}\text { Antecedent: skill and } \\
\text { consequence }\end{array}$ & $\begin{array}{l}\text { Person (speech) } \\
\text { Environment } \\
\text { Occupation (social } \\
\text { interaction) }\end{array}$ \\
\hline $\begin{array}{l}\text { Kuhn DE, Hardesty SL, } \\
\text { Sweeney NM. 2009. [45] }\end{array}$ & $\begin{array}{l}\text { Incorporated functional communication, with } \\
\text { extinction of destructive behaviour and } \\
\text { response blocking of repetitive straightening }\end{array}$ & $\begin{array}{l}\text { Antecedent: skill and } \\
\text { consequence } \\
\text { Antecedent: modifying } \\
\text { environment }\end{array}$ & $\begin{array}{l}\text { Occupation (alternative } \\
\text { replacement activity: } \\
\text { social interaction) } \\
\text { Environment (Mod) } \\
\text { Person (skills: speech) }\end{array}$ \\
\hline $\begin{array}{l}\text { Loftin RL, Odom SL, Lantz J F. } \\
\text { 2008. [46] }\end{array}$ & $\begin{array}{l}\text { Multi-component social skills intervention } \\
\text { (including peer training, social initiation } \\
\text { instruction, and self-monitoring) }\end{array}$ & $\begin{array}{l}\text { Antecedent: skill and } \\
\text { consequence }\end{array}$ & $\begin{array}{l}\text { Occupation (social } \\
\text { interaction) } \\
\text { Environment (social) } \\
\text { Person (social initiation } \\
\text { and self-monitoring) }\end{array}$ \\
\hline
\end{tabular}

Notes:

*PEO (Person Environment Occupation) Model of Occupational Performance.

${ }^{* *} \mathrm{RB}$ (Restricted Behaviour).

in the systematic review by Patterson, Smith \& Jelen [9] and some of the interventions in the individual intervention studies were found to be effective. In total twenty-eight different effective interventions were identified from eleven of the studies. Table 2 describes those studies in terms of intervention, intervention category, and PEO [6]. The Intervention category uses a classification system [7] with terms that are common within the field of $A B A$ and FBA.

\section{Data Analysis}

\section{Intervention Classification by ABA Framework}

Analysis of the total twenty-eight effective interventions named using the FBA framework proposed by Boyd et al. [7] led to nine interventions being considered within the Consequence-based intervention category, ten fell within the Antecedentbased intervention category (modifying the environment or routine to reduce the likelihood of the RRB occurring or enriching the skills of the individuals in the environment). The remaining nine interventions were found to fall under a combination of the Consequence and Antecedent categories.

\section{Intervention Classification by PEO Model}

PEO classification was determined by answering the question: Is the treatment aiming to impact the person, the environment or the occupation? Of the twenty eight effective interventions identified none were found to exclusively target the person, eleven targeted the environment only (enhancing the physical or social environment of the child), and one targeted only occupation. Many interventions targeted strategies at two or more aspects of the PEO model: two concurrently targeted the person and the environment, eleven targeted the environment and occupation and the remaining three interventions targeted all aspects of the PEO: person, environment and occupation.

A comparison of the identified effective behavioural interventions using both the ABA approach suggested by Boyd et al. [7] and the PEO model [6] can be seen in Table 3.

\section{DISCUSSION}

This integrative review explored how occupational therapists can address RRBs by incorporating known evidence-based behavioural interventions into an occupational therapy frame of reference. Analysis of the twenty-eight effective interventions identified for the treatment of RRBs in autism included in this review outlined similarities between the FBA approach suggested by Boyd et al. [7] and the PEO model [6]. The simultaneous application of these two models 
demonstrate how the interventions relate to occupational therapy theory and practice and will help guide occupational therapists in choosing the best treatment approach to use with their clients. In addition this analysis highlights for all members of interdisciplinary teams working with children with autism, the unique perspective that occupational therapists have in understanding RRBs in relation to the person, the environment, and also the occupation and how these limitations can be addressed.

\section{Interventions Targeting the Environment}

Occupational therapists often aim to impact occupational performance by changing the environment in which the occupation is performed. The Functional Behaviour Assessment when used in combination with the PEO concepts allows the therapist to consider events in the environment in terms of antecedents and consequences of behaviours and the timing of these environmental adjustments or controls. Many of the interventions (eleven) were found by the authors of this review, to be primarily targeting the environment aspect of the PEO model. Interventions that were classified under environment included consequence based interventions, antecedent based (modifying the environment or routine) interventions, as well as some antecedent based (enriching skills) interventions.

An example of an intervention that targeted change to the environment by changing or improving the skills of others in the child's world is the Family-Implemented Treatment for Behavioural Inflexibility (FITBI) [41]. A parent-group intervention in which the parents were trained and offered new skills in order to address their child's RRBs. This approach would be seen as changing the antecedent or what happens before the behaviour occurs by enriching the skills of individuals in the child's environment and by doing so affecting the RRB.

Some interventions targeted the environment by modifying the physical surroundings in which the behaviour was performed. Examples of such interventions include the use of visual schedules or video based technologies $[27,28]$. Using the FBA framework, these strategies are examples of modifying the environment as an antecedent approach. Other interventions used the environment as a consequence strategy by changing the environment after the RRB occurred to either reinforce or deter the performance of the RRB. Finally, a few interventions utilized the environment as both an antecedent and a consequence strategy by differential reinforcement with and without extinction [14, 18, 34].

\section{Interventions Targeting the Person}

In practice the occupational therapist may address the individual's skills and determine if they fit with the occupation possibly having an impact on occupational performance. If the person's skills do not match the demands of the occupation and the environment where the task is being performed, intervention at the skill level of the person will need to be addressed. Of all the effective interventions identified in this study, none were found to target solely the person level of the PEO model exclusively.

\section{Interventions Targeting Occupation}

Occupational therapists also influence changes at the level of the occupation as a way to impact occupational performance or behaviour. "Occupations refer to the everyday activities that people do as individuals, in families and with communities to occupy time and bring meaning and purpose to life. Occupations include things people need to, want to and are expected to do" [5]. When considering the number of effective treatment options discovered in the literature, the occupational therapist must consider how "occupation" fits.

Occupation may be incorporated into intervention for RRBs as an antecedent event that will decrease the likelihood of a problematic behaviour such as RRB occurring. Kern, Koegel, \& Dunlap [32] identified one intervention that used the occupation of physical exercise in this way. By exercising, the person is engaging in a functional occupation that may fulfill the same sensory needs as the RRB. Exercise therefore eliminates the need for engagement in the RRB and serves as a replacement behaviour for the nonfunctional RRB. The type of antecedent occupation that is facilitated however will be dependent on the person and therefore must be chosen and/or adapted based on the needs and abilities of the person.

\section{Interventions Targeting Person and Environment}

A few interventions identified addressed both the person and the environment aspects of the PEO model. These interventions included antecedent (enrichment of skills) approaches, antecedent (modifying the environment) approaches, as well as consequence based approaches. 
Table 3: RRB Intervention Classification and Comparison between the ABA approach and the PEO Model of Occupational Performance

\begin{tabular}{|c|c|c|c|c|}
\hline & \multicolumn{4}{|c|}{ Functional Behaviour Analysis Concepts } \\
\hline & $\begin{array}{l}\text { Antecedent based } \\
\text { Interventions } \\
\text { (Modifying environment } \\
\text { or routine) }\end{array}$ & $\begin{array}{l}\text { Antecedent } \\
\text { Based Interventions } \\
\text { (Enriching Skills) }\end{array}$ & $\begin{array}{c}\text { Consequence } \\
\text { Based Interventions }\end{array}$ & $\begin{array}{l}\text { Consequence and } \\
\text { Antecedent Based } \\
\text { interventions }\end{array}$ \\
\hline \multicolumn{5}{|l|}{ PEO Components } \\
\hline Person & NA & NA & NA & NA \\
\hline \multirow[t]{6}{*}{ Environment } & $\begin{array}{c}\text { Visual schedules or video } \\
\text { based technologies } \\
{[27,28]}\end{array}$ & $\begin{array}{l}\text { Family-implemented } \\
\text { treatment for behavioural } \\
\text { inflexibility [41] }\end{array}$ & $\begin{array}{l}\text { Differential reinforcement of } \\
\text { variability }[17,18]\end{array}$ & $\begin{array}{l}\text { Exposure response } \\
\text { Prevention [42] }\end{array}$ \\
\hline & & & $\begin{array}{l}\text { Response cost procedures } \\
\qquad[12,13]\end{array}$ & * \\
\hline & $\begin{array}{l}\text { Visual or verbal } \\
\text { Cues[19,20] }\end{array}$ & $\begin{array}{c}\text { Managing } \\
\text { Repetitive } \\
\text { Behavior Program[43] }\end{array}$ & $\begin{array}{l}\text { Tangible reinforce } \\
\qquad[44]\end{array}$ & \\
\hline & & & Social praise [44] & \\
\hline & & & $\begin{array}{l}\text { Differential reinforcement } \\
\text { plus extinction [34] }\end{array}$ & \\
\hline & & & $\begin{array}{c}\text { Differential Reinforcement } \\
\text { [14] }\end{array}$ & \\
\hline Occupation & Physical exercise [32] & & & \\
\hline \multicolumn{5}{|c|}{ PEO Component Interactions } \\
\hline $\begin{array}{l}\text { Person and } \\
\text { Environment }\end{array}$ & & $\begin{array}{c}\text { Cognitive Behavioural } \\
\text { Therapy (parents and } \\
\text { child) [49] }\end{array}$ & & $\begin{array}{c}\text { Cognitive behavioural } \\
\text { therapy plus exposure } \\
\text { response prevention } \\
{[29,30]}\end{array}$ \\
\hline \multirow[t]{4}{*}{$\begin{array}{l}\text { Occupation and } \\
\text { Environment. }\end{array}$} & $\begin{array}{c}\text { Antecedent based uses } \\
\text { of circumscribed interests } \\
{[24,25]}\end{array}$ & & $\begin{array}{c}\text { Consequence based use of } \\
\text { circumscribed interests } \\
{[15,16]}\end{array}$ & $\begin{array}{l}\text { Response interruption } \\
\text { redirection plus matched } \\
\text { stimulus [3] }\end{array}$ \\
\hline & $\begin{array}{c}\text { Environmental } \\
\text { enrichment strategies } \\
{[21,22,23]}\end{array}$ & & $\begin{array}{c}\text { Response interruption and } \\
\text { redirection/response blocking } \\
{[9,10,11]}\end{array}$ & $\begin{array}{l}\text { Matched stimulation and } \\
\text { noncontingent } \\
\text { reinforcement [39] }\end{array}$ \\
\hline & $\begin{array}{l}\text { Noncontingent access to } \\
\text { matched stimuli [3] }\end{array}$ & & $\begin{array}{l}\text { Response interruption and } \\
\text { redirection }[3,9]\end{array}$ & $\begin{array}{l}\text { Matched item } \\
\text { plus blocking [48] }\end{array}$ \\
\hline & $\begin{array}{c}\text { Noncontingent access } \\
{[38]}\end{array}$ & & & $\begin{array}{c}\text { Matched item plus } \\
\text { prompts plus blocking } \\
{[48]}\end{array}$ \\
\hline \multirow{3}{*}{$\begin{array}{l}\text { Occupation, } \\
\text { Environment, and } \\
\text { Person }\end{array}$} & & & & $\begin{array}{l}\text { Multi-component social } \\
\text { skills intervention [46] }\end{array}$ \\
\hline & & & & $\begin{array}{l}\text { Functional } \\
\text { communication training } \\
(\text { FCT) with } \\
\text { reinforcement [31] }\end{array}$ \\
\hline & & & & $\begin{array}{c}\text { Functional } \\
\text { communication plus } \\
\text { extinction of destructive } \\
\text { behaviour plus response } \\
\text { blocking [45] }\end{array}$ \\
\hline
\end{tabular}


Cognitive behaviour therapy (CBT) [29] targets the cognitive skills of the individual while also targeting change in the environment by using exposure and response prevention at the same time. Not only were cognitive reframing skills taught to the client, they were physically applied using strategies that involved access or denial of items in the environment that were related to the RRBs.

\section{Interventions Targeting Occupation and Environment}

Some interventions targeted occupation while also targeting the environment. An example includes matched Item, plus blocks and prompting approach [48]. In this approach the performance of RRB is blocked and participation in use of the matched item is prompted. This intervention is both antecedent type (modifying the environment) and consequence type.

\section{Interventions Targeting Occupation, Environment, and Person}

Functional communication training (FCT) [45] addresses person, occupation and environment. The person component is addressed as improving skills (speaking) and the occupation of social interaction is done through modification of the task by providing prompts. The environment (modifying) is involved through the availability of items dependent upon whether RRB are occurring or not. Treatments are considered to be antecedent (enhancing skills of client or others in the child's environment) and consequence based (access or denial of reinforcers).

\section{Occupational Therapy and Occupation as Part of Intervention for RRB}

Since occupational therapists aim to make change in occupational performance or behaviour by influencing change in any or all aspects of the PEO Model, interventions that target change under any of these categories are within the occupational therapy scope of practice.

Occupation has been incorporated into RRB interventions through the use of the alternative replacement behaviour - typically recommended as part of a FBA. The alternative replacement behaviour is considered the functional equivalent to the identified problematic behaviour [51]. The alternative replacement behaviour is a new behaviour, chosen by the therapist that is more appropriate or more functional than the problematic behaviour. To occupational therapists, this alternative replacement behaviour may be seen as the modified occupation; the occupation is changed in such a way that it will enable successful occupational performance. The alternative replacement behaviour is maintained by the same consequence that maintained the problematic behaviour or RRB. In other words it provides the same reinforcement that the problematic behaviour provided. The function of RRBs for individuals with autism has frequently been determined to be a need for unique sensory input [52]. Boyd et al., [7] discuss how this can sometimes complicate the development of interventions that target repetitive behaviour since understanding what the child actually gains or escapes by engaging in the sensory behaviour can be difficult. In relation to treatment for RRBs, Patterson with Smith \& Jelen (2010) [33] suggest "The clinical expertise of occupational therapists who work with individuals with ASD may prove to be a valuable resource in the decision making process" (p. 325) for this reason.

Occupational therapists are skilled in the assessment of occupational performance by understanding occupation or task requirements, the influences of the environment (physical, social, sensory as antecedents and consequences) and the skills of the person (cognitive, perceptual, physical, sensory) and then determining how those components match or fit together. If there is not a good fit resulting in occupational dysfunction, the occupational therapist may modify the environment, the occupation, or enhance the individual's skills as part of treatment in order to achieve optimal occupational performance. Occupational Therapists may therefore be uniquely qualified in determining the maintaining consequences of a problematic behaviour and also in recommending appropriate alternative replacement behaviours/occupations that would result in the same consequences as the RRB, particularly those that seem to be related to a sensory need.

\section{LIMITATIONS}

It is possible that all known evidenced based interventions identified to effectively address RRBs were not identified as a result of the search completed for this review based upon the search terms utilized.

The classification of the evidenced based interventions identified according to the PEO Model was based on the opinion and analysis of the author group. Other therapists or professionals may view the intervention categorization differently. The same is true 
for the classification of identified interventions according to the FBA framework. Many were classified previously by the author Boyd et al. [7] however the remaining interventions that were not part of that study were classified based on the opinion of the author of the present study as well. Nonetheless, the similarities between how the two frameworks align could be useful to the occupational therapist in determining the intervention approach for their respective client needs.

The current analysis is not specific in terms of RRBs type and the corresponding effective evidenced based intervention(s) (e.g., effective interventions for the RRB of spinning repetitively). In addition effective interventions for RRBs for individuals with and without intellectual disabilities were not addressed separately. Further delineation of interventions for specific RRB, together with addressing the potential for the interaction with the level of intellectual ability is warranted.

\section{CONCLUSION}

Occupational Therapists have an important role to play in the treatment of RRBs seen in individuals diagnosed with autism. As members of interdisciplinary teams working with this population, occupational therapists can enable improved engagement and performance in meaningful occupations in part by decreasing RRBs and teaching new skills. This can be achieved in many ways by targeting various or multiple aspects of the PEO model which map well onto the list of interventions identified in this review. This review provides a framework for occupational therapists to understand and utilize behavioural interventions in the context of occupational performance. This new information will also inform other professionals of the potential role the occupational therapists may have with this population.

\section{REFERENCES}

[1] American Psychological Association. Diagnostic and statistical manual of mental disorders. 5th ed. Arlington, VA: American Psychiatric Publishing 2013.

[2] Lam KS, Aman MG. The repetitive behaviour scale - revised: independent validation in individuals with autism spectrum disorders. J Autism Dev Disord 2007; 37: 855-866. https://doi.org/10.1007/s10803-006-0213-z

[3] Love JJ, Miguel CF, Fernand JK, LaBrie JK. The effects of matched stimulation and response interruption and redirection on vocal stereotypy. J Appl Behav Anal 2012; 45: 549-564. https://doi.org/10.1901/jaba.2012.45-549

[4] World Federation of Occupational Therapists (WFOT). Definition of occupational therapy: statement of occupational therapy. 2012; Available at: http://www.wfot.org/AboutUs/
AboutOccupationalTherapy/DefinitionofOccupationalTherapy .aspx. Accessed Dec 3, 2016.

[5] World Federation of Occupational Therapists (WFOT) Definition of occupational therapy: Definition "Occupation." Available at: http://www.wfot.org/aboutus/aboutoccupationaltherapy/definitionofoccupationaltherapy.aspx. Accessed Dec 4, 2016.

[6] Law M, Cooper BA, Strong S, Stewart D, Rigby P, Letts L. The Person-Environment-Occupational Model: a transactive approach to occupational performance. Can J Occup Ther 1996; 63(1): 9-23. https://doi.org/10.1177/000841749606300103

[7] Boyd BA, Mcdonough SG, Bodfish JW. Evidence-based behavioural interventions for repetitive behaviours in autism. J Autism Dev Disord 2012; 42(6): 1236-48. Boyd BA, McDonough SG, Bodfish JW.

[8] Whittemore R, Knafl K. The integrative review: updated methodology. J Adv Nurs 2005; 52: 546-553. https://doi.org/10.1111/j.1365-2648.2005.03621.x

[9] Ahearn WH, Clark KM, Macdonald RPF, Chung BI. Assessing and treating vocal stereotypy in children with autism. J Appl Behav Anal 2007; 40(2): 263-275. https://doi.org/10.1901/jaba.2007.30-06

[10] Koegel RL, Firestone PB, Kramme KW, Dunlap G. Increasing spontaneous play by suppressing self- stimulation in autistic children. J Appl Behav Anal 1974; 7(4): 521-528. https://doi.org/10.1901/jaba.1974.7-521

[11] Liu-Gitz L, Banda DR. A replication of the RIRD strategy to decrease vocal stereotypy in a student with autism. Behavioural Interventions 2010; 25(1): 77-87.

[12] Athens E. S, Vollmer TR, Sloman KN, Pipkin CSP. An analysis of vocal stereotypy and therapist fading. J Appl Behav Anal 2008; 41(2): 291-297.

https://doi.org/10.1901/jaba.2008.41-291

[13] Sidener TM, Carr JE, Firth AM. Superimposition and withholding of edible consequences as treatment for automatically reinforced stereotypy. J Appl Behav Anal 2005; 38(1): 121-124.

https://doi.org/10.1901/jaba.2005.58-04

[14] Azrin NH, BesaleIV, Jamner JP, Caputa JN. Comparative study of behavioral methods of treating severe self-injury. Behavior Resident Treatment 1988; 3: 119-152. https://doi.org/10.1002/bin.2360030204

[15] Charlop-Christy M, Haymes L. Using obsessions as reinforcers with and without mild reductive procedures to decrease inappropriate behaviours of children with autism. J Autism Dev Disord 1996; 26(5): 527-546. https://doi.org/10.1007/BF02172274

[16] Charlop-Christy M, Haymes L. Using objects of obsession as token reinforcers for children with autism. J Autism Dev Disord 1998; 28(3): 189-198. https://doi.org/10.1023/A:1026061220171

[17] Boyd BA, Baranek GT, Sideris J, Poe M, Watson LR, Patten $\mathrm{E}$, et al. Sensory features and repetitive behaviors in children with autism and developmental delays. Autism Research 2010; 3: 78-87.

https://doi.org/10.1002/aur.124

[18] Miller N, Neuringer A. Reinforcing variability in adolescents with autism. J Appl Behav Anal 2000; 33(2): 151-165. https://doi.org/10.1901/jaba.2000.33-151

[19] Conroy MA, Asmus JM, Sellers JA, Ladwig CN. The use of an antecedent- based intervention to decrease stereotypic behaviour in a general education classroom: A case study. Focus Autism Other Dev Disabl 2005; 20(4): 223-230. https://doi.org/10.1177/10883576050200040401

[20] Horner RH, Day HM, Day JR. Using neutralizing routines to reduce problem behaviors. J Appl Behav Anal 1997; 30: 601614. https://doi.org/10.1901/jaba.1997.30-601 
[21] Piazza CC, Adelinis JD, Hanley GP, Goh H, Delia MD. An evaluation of the effects of matched stimuli on behaviours maintained by automatic reinforcement. J Appl Behav Anal 2000; 33(1): 13-27.

https://doi.org/10.1901/jaba.2000.33-13

[22] Rapp JT, Vollmer, Timothy R. Stereotypy I: A review of behavioural assessment and treatment. Research in Developmental Disabilities: A Multidisciplinary Journal 2005; 26(6): 527-547.

https://doi.org/10.1016/j.ridd.2004.11.005

[23] Vollmer TR, Others A. Treatment of self- injury and hand mouthing following inconclusive functional analyses. J Appl Behav Anal 1994; 27(2): 331-44.

https://doi.org/10.1901/jaba.1994.27-331

[24] Baker MJ. Incorporating the thematic ritualistic behaviors of children with autism into games: Increasing social play interactions with siblings. J Posit Behav Interv 2000; 2(2): 6684.

https://doi.org/10.1177/109830070000200201

[25] Baker MJ, Koegel RL, Koegel LK. Increasing the social behavior of young children with autism using their obsessive behaviors. J Assoc Pers Sev Handicaps 1998; 23: 300-308. https://doi.org/10.2511/rpsd.23.4.300

[26] Boyd BA, Conroy MA, Mancil GR, Nakao T, Alter PJ. Effects of circumscribed interests on the social behaviours of children with autism spectrum disorders. J Autism Dev Disord 2007; 37(8): 1550-1561.

https://doi.org/10.1007/s10803-006-0286-8

[27] Hine JF, Wolery M. Using point-of- view video modeling to teach play to preschoolers with autism. Topics in Early Childhood Special Education 2006; 26(2): 83-93 https://doi.org/10.1177/02711214060260020301

[28] Odom SL, Brown WH, Frey T, Karasu N, Smith-Canter L, Strain PS. Evidence- based practices for young children with autism: Contributions for single-subject design research. Focus Autism Other Dev Disabl 2003; 18(3): 166-75. https://doi.org/10.1177/10883576030180030401

[29] Lehmkuhl HD, Storch EA, Bodfish JW, Geffken GR. Brief report: Exposure and response prevention for obsessive compulsive disorder in a 12-year-old with autism. J Autism Dev Disord 2008; 38(5): 977-981. https://doi.org/10.1007/s10803-007-0457-2

[30] Reaven J, Hepburn S. Cognitive- behavioural treatment of obsessive- compulsive disorder in a child with Asperger syndrome: A case report. Autism 2003; 7(2): 145. https://doi.org/10.1177/1362361303007002003

[31] Kennedy CH, Meyer KA, Knowles T, Shukla S. Analyzing the multiple functions of stereotypical behaviour for students with autism: Implications for assessment and treatment. J Appl Behav Anal 2000; 33(4): 559-571. https://doi.org/10.1901/jaba.2000.33-559

[32] Kern L, Koegel R, Dunlap G. The influence of vigorous versus mild exercise on autistic stereotyped behaviours. J Autism Dev Disord 1984; 14(1): 57-67. https://doi.org/10.1007/BF02408555

[33] Patterson SY, Smith V, Jelen M. Behavioural intervention practices for stereotypic and repetitive behaviour in individuals with autism spectrum disorder: A systematic review. Dev Med Child Neurol 2010; 52(4): 318-327. https://doi.org/10.1111/j.1469-8749.2009.03597.x

[34] Rehfeldt RA, Chambers MR. Functional analysis and treatment of verbal perseverations displayed by an adult with autism. J Appl Behav Anal 2003; 36(2): 259-261. https://doi.org/10.1901/jaba.2003.36-259

[35] Tarbox J, Wallace MD, Tarbox RSF. Successful generalized parent training and failed schedule thinning of response blocking for automatically maintained object mouthing. Behavioural Interventions 2002; 17(3): 169-178. https://doi.org/10.1002/bin.116
[36] Carr JE, Dozier CL, Patel MR, Adams AN, Martin N. Treatment of automatically reinforced object mouthing with noncontingent reinforcement and response blocking: Experimental analysis and social validation. Res Dev Disabil 2002; 23(1): 37-44. https://doi.org/10.1016/S0891-4222(01)00090-7

[37] Cicero FR. The effects of noncontingent reinforcement and response interruption on stereotypic behavior maintained by automatic reinforcement [dissertation]. New York: City: University of New York 2007.

[38] Roane HS, Kelly ML, Fisher WW. The effects of noncontingent access to food on the rate of object mouthing across three settings. J Appl Behav Anal 2003; 36(4): 579582. https://doi.org/10.1901/jaba.2003.36-579

[39] Rapp JT. Further evaluation of methods to identify matched stimulation. J Appl Behav Anal 2007; 40(1): 73-88. https://doi.org/10.1901/jaba.2007.142-05

[40] Britton LN, Carr JE, Landaburu HJ, Romick KS. The efficacy of noncontingent reinforcement as treatment for automatically reinforced stereotypy. Behavioral Interventions 2002; 17(2): 93-103. https://doi.org/10.1002/bin.110

[41] Boyd BA, McDonough SG, Rupp B, Khan F, Bodfish JW. Effects of a family-implemented treatment on the repetitive behaviors of children with autism. Journal of Autism and Developmental Disorders 2011; 41(10): 1330-1341. https://doi.org/10.1007/s10803-010-1156-y

[42] Boyd BA, Woodard CR, Bodfish JW. Feasibility of exposure response prevention to treat repetitive behaviors of children with autism and an intellectual disability: A brief report. Autism: The International Journal of Research and Practice 2013; 17(2): 196-204. https://doi.org/10.1177/1362361311414066

[43] Grahame V, Brett D, Dixon L, McConachie H, Lowry J, Rodgers $\mathrm{J}$, et al. Managing repetitive behaviours in young children with autism spectrum disorder (ASD): Pilot randomised controlled trial of a new parent group intervention. J Autism Dev Disord 2015; 45(10): 3168-3182. https://doi.org/10.1007/s10803-015-2474-x

[44] Kang S, O'Reilly M, Rojeski L, Blenden K, Xu Z, Davis T, et al. Effects of tangible and social reinforcers on skill acquisition, stereotyped behavior, and task engagement in three children with autism spectrum disorders. Research in Developmental Disabilities: A Multidisciplinary Journal 2013; 34(2): 739-744 https://doi.org/10.1016/j.ridd.2012.10.007

[45] Kuhn DE, Hardesty SL, Sweeney NM. Assessment and treatment of excessive straightening and destructive behavior in an adolescent diagnosed with autism. J Appl Behav Anal 2009; 42(2): 355-360. https://doi.org/10.1901/jaba.2009.42-355

[46] Loftin RL, Odom SL, Lantz JF. Social interaction and repetitive motor behaviors. J Autism Dev Disord 2008; 38(6): 1124-1135.

https://doi.org/10.1007/s10803-007-0499-5

[47] Murdock LC, Dantzler JA, Walker AN, Wood LB. The effect of a platform swing on the independent work behaviors of children with autism spectrum disorders. focus on autism and other developmental disabilities 2014; 29(1): 50-61. https://doi.org/10.1177/1088357613509838

[48] Rodriguez NM, Thompson RH, Stocco CS, Schlichenmeyer $\mathrm{K}$. Arranging and ordering in autism spectrum disorder: Characteristics, severity, and environmental correlates. Journal of Intellectual \& Developmental Disability 2013; 38(3): 242.

https://doi.org/10.3109/13668250.2013.812192

[49] Storch EA, Arnold EB, Lewin AB, Nadeau J, Jones AM, De Nadai AS, et al. Effect of cognitive- behavioral therapy versus treatment as usual for anxiety in children with autism 
spectrum disorders: A randomized, controlled trial. Journal of the American Academy of Child \& Adolescent Psychiatry 2012.

[50] Watling R, Deitz J, Kanny EM, McLaughlin JF. Current practice of occupational therapy for children with autism. American Journal of Occupational Therapy 1999; 53(5): 498505.

https://doi.org/10.5014/ajot.53.5.498
[51] Carr EG, Durand VM. Reducing behaviour problems through functional communication training. J Appl Behav Anal 1985; 18(2): 111-126.

https://doi.org/10.1901/jaba.1985.18-111

[52] Iwata BA, Others A. Toward a functional analysis of selfinjury. J Appl Behav Anal 1994; 27(2): 197-209. https://doi.org/10.1901/jaba.1994.27-197

DOI: https://doi.org/10.6000/2292-2598.2017.05.03.1

(c) 2017 Patriquin et al.; Licensee Lifescience Global.

This is an open access article licensed under the terms of the Creative Commons Attribution Non-Commercial License (http://creativecommons.org/licenses/by-nc/3.0/) which permits unrestricted, non-commercial use, distribution and reproduction in any medium, provided the work is properly cited. 